\title{
Statistical Cerebrovascular Segmentation in Three- Dimensional Rotational Angiography Based on Maximum Intensity Projections
}

\author{
Rui Gan ${ }^{\mathrm{a}}{ }^{*}$, Wilbur C. K. Wong ${ }^{\mathrm{a}}$, \\ Albert C. S. Chung a and Simon C. H. Yu ${ }^{\mathrm{b}}$ \\ ${ }^{a}$ Department of Computer Science, Hong Kong University of Science and Technology, Hong Kong. \\ ${ }^{b}$ Department of Diagnostic Radiology and Organ Imaging, Prince of Wales Hospital, Hong Kong.
}

\begin{abstract}
Vascular segmentation of three-dimensional rotational angiography (3D-RA) is important in the clinical environment since it can provide 3D information of vasculature before, during and after the treatments. This paper extends our prior work on vascular segmentation method for 3D-RA, which is based on maximum intensity projections (MIP). The method is fully automatic and computationally efficient. Experimental results on 12 3D-RA clinical datasets indicate that our method can produce segmentations of major vessels in the datasets, which are the current radiologists' primary interest in this work. Moreover, the segmentations obtained by our method exhibit a high degree of agreement to the ground truth segmentations and are comparable to those produced by the optimal global thresholding method.
\end{abstract}

Keywords: Image segmentation; 3D rotational angiography (3D-RA); maximum intensity projection (MIP); expectation maximization (EM) algorithm; finite mixture model (FMM).

\section{Introduction}

Three-dimensional rotational angiography (3D-RA) is a relatively new technique for imaging blood vessels, and is widely used in neuroradiological interventions [1]. A 3D$\mathrm{RA}$ image volume is reconstructed from a sequence of 2D low dose rotational X-ray angiograms acquired at different viewing angles. The new modality can provide morphological information of the cerebral vessels during operations and is a very useful imaging tool for assessing intracranial aneurysms and arteriovenous malformations $(\mathrm{AVM})$ in endovascular treatments.

In this paper, we extend our prior work on vascular segmentation method for 3D-RA [2], which is based on maximum intensity projections (MIP). MIP is the simplest volume rendering technique and is commonly used by radiologists to visualize $3 \mathrm{D}$ vascular morphology on a 2D image (i.e. the MIP image) [3]. The proposed segmentation technique is a statistical approach based on the finite mixture model (FMM) and the expectation maximization (EM) algorithm [4] to segmenting 3D-RA image volumes. It

\footnotetext{
* Corresponding author. E-mail address: raygan@cs.ust.hk.
} 
exploits a property of MIP, namely, the proportion of vessels in the MIP image is large as compared with the original 3D volume, to increase the stability in the parameter estimation of FMM with the EM algorithm. Our method takes an iterative approach to segmenting 3D-RA images progressively with the segmentation of MIP images along the three principle axes. Moreover, the proposed method is fully automatic and computationally efficient.

We have tested our segmentation technique on 12 3D-RA clinical datasets. The experimental results indicate that the automatic method can produce segmentations of major vessels in the datasets, which are the current radiologists' primary interest in this work. Moreover, the segmentations obtained by our method exhibit a high degree of agreement to the manual segmentations obtained by an experienced consultant radiologist in a local hospital (we treat the manual segmentations as the ground truth). In a further comparison with the global thresholding method, we found that the vascular segmentation from our algorithm is comparable to the one produced by the optimal global threshold.

\section{Problem statement}

Vascular segmentation of 3D-RA is important in the clinical environment to provide 3D vascular information before, during and after the endovascular treatments. Global thresholding method is a practical and efficient approach to 3D-RA segmentation since it can produce 3D segmentation in seconds with a manually selected or a pre-defined threshold. According to radiologists' feedback, the segmentation of 3D-RA with a global threshold is good enough for most of the clinical applications because vessels are enhanced by the use of contrast agent. However, owing to the relative large variance in the intensity values of vessels amongst different 3D-RA images, it is necessary for a radiologist to select the global threshold manually for each 3D-RA image in order to produce adequate vascular segmentation. The manual threshold selection process can be very subjective and it is of low repeatability. Therefore, an automatic threshold selection method is preferable in practice.

FMM and the EM algorithm, based on the maximum likelihood (ML) estimation, provide means to the automatic global threshold selection, as have been demonstrated in [5]. Because of the implementation simplicity and computational efficiency of the approach, segmentation methods based on FMM and the EM algorithm, which is known as the EM-based segmentation method (hereafter referred to as Auto-GT), are very attractive to the time-critical medical applications. In our previous work [2], we found that a double-Gaussian mixture model can be adopted to estimate the intensity distribution of 3D-RA images, where the Gaussian function with a higher mean represents the intensity distribution of vessels in 3D-RA images and the other one (i.e. with a lower mean) corresponds to the intensity distribution of the background.

However, in the experiments on 12 3D-RA clinical datasets, we observe that it can be very difficult to accurately model the intensity distribution of vessels in 3D-RA with a Gaussian distribution. This is because, in a typical 3D-RA image, the volume occupied by vessels is very small (on average, $<2 \%$ of the total volume). Therefore, the Gaussian distribution with a higher mean in the mixture model may shift to the low intensity range 
in the parameter estimation process (via the EM algorithm). As a result, a relatively low threshold is selected and the 3D-RA images are over segmented.

\section{Method}

The proposed method is based on a property of MIP, namely, the larger proportion of vessels in the MIP image as compared with that in the original 3D volume, and it compiles the vascular segmentation iteratively through the segmentation of MIP images projected along a fixed direction. Our method begins with generating an MIP image from the original 3D volume. In order to avoid unnecessary intensity interpolation, we select the projection direction to one of the three principle axes.

At each iteration, an MIP image is generated from the 3D volume, while the 3D position of the voxels that contributed to the MIP image is recorded. The MIP image is then segmented by Auto-GT. In this paper, as compared with our previous work [2], a much simpler mixture model, i.e. a double-Gaussian model, is used for approximating the intensity distribution of the MIP image, which can make the parameter estimation of FMM with the EM algorithm more robust. The intensity distribution of vessel class is modeled by the Gaussian function with a larger mean, while, that of background class is modeled by the other Gaussian function. The voxels corresponding to vessel pixels in the MIP image are added to the 3D vascular segmentation. Those voxels are then removed from the 3D volume and will not contribute to the MIP image generation at the next iteration. The algorithm continues to iterate until no pixels are classified as vessel in the MIP image.

It is noted that, at each successive iteration, more vessel voxels are added to the $3 \mathrm{D}$ vascular segmentation and removed from the 3D volume for the exclusion in the maximum intensity projection process. Due to the significant decrease in the number of high intensity pixels (i.e. the number of vascular pixels) in the subsequent MIPs, the Gaussian distribution with a higher mean in the mixture model may shift to the lower intensity region. A relatively low threshold is then selected to segment the MIP image and as a result over segmentation is produced. Therefore, we have proposed a novel mechanism in our previous work [2], which can automatically detect the left-shift of the aforesaid Gaussian distribution and the termination of our algorithm.

For a further illustration, suppose that the current iteration is $k$. The proposed mechanism evaluates the difference between two sums of absolute difference (SAD), which are respectively between the current background intensity distribution $h_{k}^{B}$ (it can be estimated from the threshold calculated at the previous iteration), and two distributions estimated via the EM algorithm: the first Gaussian distribution in the mixture model, $h_{k}^{G}$, and the overall double-Gaussian distribution, $h_{k}^{G G}$. If the Gaussian distribution with a higher mean is left-shifted, it is expected to have a smaller SAD value between $h_{k}^{B}$ and $h_{k}^{G G}$ than the SAD value between $h_{k}^{B}$ and $h_{k}^{G}$. This is because $h_{k}^{G G}$ gives a better approximation on $h_{k}^{B}$ when the aforesaid Gaussian distribution is shifted to the low intensity range. Therefore, the stopping criterion of our algorithm is to see if $S A D\left(h_{k}^{B}, h_{k}^{G}\right)>S A D\left(h_{k}^{B}, h_{k}^{G G}\right)$. 
Once the stopping criterion is satisfied, the algorithm stops generating MIP images and gives the compiled $3 \mathrm{D}$ vascular segmentation as the final segmentation. However, it does not imply that no vessel voxel can be found in the rest of the 3D volume. As such, in order not to leave out any vessel voxels, we apply the threshold selected at the second last iteration to segment the rest of the $3 \mathrm{D}$ volume.

For increasing the accuracy and robustness of our method and decrease the viewingangle dependency, we propose a proper extension of the aforesaid algorithm to exploit the segmentation of MIP images projected along the three principles axes. The extension can be outlined as follows: (1) we execute the above segmentation algorithm with MIP images generated in different projection directions, i.e. along $\mathrm{X}$-axis, Y-axis and Z-axis, and (2) we combine the three segmentations under an aggregation scheme to derive the final vascular segmentation. In this paper, unlike the aggregation method based on energy optimization in our previous work [2], which is more computational expensive, we use a winner-takes-all (WTA) based method for combining the three segmentations without degradation of the final segmentation result.

Our purposed vascular segmentation method can be summarized as follows: (1) along the three principle axes, we execute the aforementioned segmentation method to obtain the three segmentations separately and (2) aggregate the three segmentations under the WTA scheme to derive the final vascular segmentation.

\section{Results}

We have applied the proposed method on 12 3D-RA clinical datasets acquired by Philips Integris imager at the Department of Diagnostic Radiology and Organ Imaging, Prince of Wales Hospital (PWH), Hong Kong. The data volume is around $100 \times 100 \times 100$ voxels with a voxel size of $0.75 \times 0.75 \times 0.75 \mathrm{~mm}^{3}$. Furthermore, the 12 datasets are manually segmented by an experienced consultant radiologist at $\mathrm{PWH}$, who has more than 15 years' clinical experience in endovascular neurosurgery. The manual segmentations are treated as the ground truth in the experiments.

In order to study the segmentation accuracy of the proposed method, we have applied Auto-GT and our novel method (hereafter referred to as MIP-MP-WTA) to the 12 3DRA clinical datasets for comparison. All the obtained segmentations are compared based on Dice similarity coefficient (DSC) [6], which can take both the sensitivity and specificity into account in the measurement of agreement, towards the ground truth (i.e. the manual segmentations).

Fig. 1 presents the DSC values for Auto-GT, MIP-MP-WTA and the segmentation produced by the optimal global thresholding method (hereafter referred as to Opt-GT). The optimal threshold is an intensity value that can produce segmentation with the global thresholding method in maximum agreement (measured by DSC) to the ground truth. From the figure, it is indicated that our novel segmentation method (MIP-MPWTA) consistently produces segmentations in a high degree of agreement to the ground truth. On the contrary, Auto-GT gives less than satisfactory segmentations and also a large variation of the DSC values in the datasets. We also found that the DSC values for MIP-MP-WTA and Opt-GT across different clinical datasets are very close to each other. This suggests that MIP-MP-WTA is capable of producing segmentations of high 
quality that are comparable to those obtained from the consultant radiologist and OptGT.
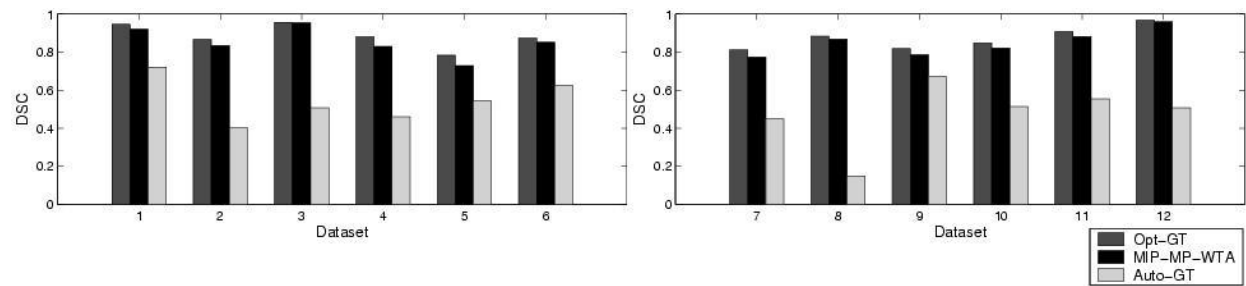

Fig. 1. The DSC values across 12 3D-RA clinical datasets for Auto-GT, MIP-MP-WTA and the segmentation produced by the optimal global thresholding method (i.e. Opt-GT).

Fig. 2 shows the regions of interests (ROI) of an image slice from a 3D-RA clinical dataset. In the figure, subfigure (a) shows the image of the ROI, subfigure (b) shows the manual segmentation produced by a local experienced consultant radiologist (i.e. ground truth), subfigures (c), (d) and (e) show the segmentations obtained by Opt-GT, Auto-GT and MIP-MP-WTA respectively. It is observed that the segmentations obtained by OptGT and MIP-MP-WTA are very similar to manual segmentations. Amongst the segmentations shown in subfigures (b), (c) and (e), there are discrepancies in only a few voxels. Contrary to our segmentations, the segmentations obtained by Auto-GT are less than satisfactory. The vascular segmentations include part of the dim non-vascular structures in the background, as shown in subfigure (d).

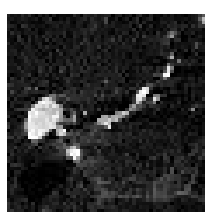

(a)

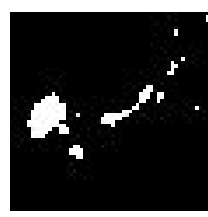

(b)

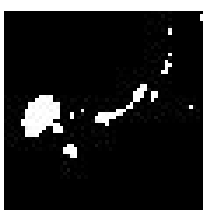

(c)

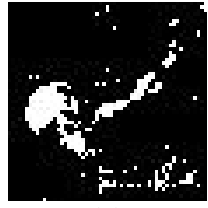

(d)

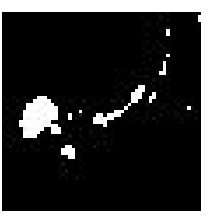

(e)

Fig. 2. ROI of an image slice from a 3D-RA clinical dataset. (a) The image of the ROI. Segmentation by: (b) a local experienced consultant radiologist (i.e. ground truth), (c) Opt-GT, (d) Auto-GT and (e) MIP-MP-WTA.

Fig. 3 shows volume rendered images of a 3D-RA dataset. Subfigure (a) show the axial MIP image, subfigures (b), (c) and (d) show the volume rendered images based on the segmentations produced by the consultant radiologist, Auto-GT and MIP-MP-WTA respectively. Volume rendering is performed by the Visualization Toolkit (VTK). It is very clear from the figure that our vascular segmentation technique is capable of segmenting 3D-RA automatically with a very high degree of agreement to the manual segmentations. Although, as shown in Fig. 3(d), it is observed that there are some nonvascular structures included in our segmentation, they can be removed automatically by a connectivity filter. It is expected that the overall quality of the segmentations will not be affected $^{1}$ after the application of the connectivity filter. This is because these nonvascular structures included in our segmentations are sparse and highly isolated from the major vascular structures, as opposed to those obtained by Auto-GT where the non-

${ }^{1}$ It is noted that the segmentation of major vessels is the current radiologists' primary interest in this work. 
vascular structures are attached to the major vessels (for better illustrations, see Fig. 2(d)).

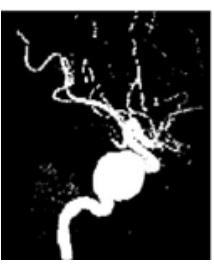

(a)

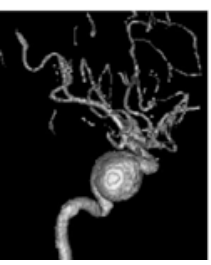

(b)

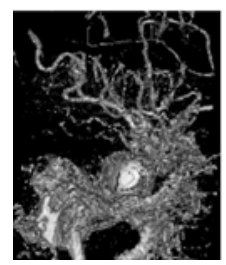

(c)

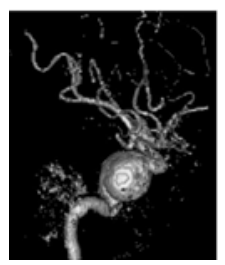

(d)

Fig. 3. Volume rendered images of a 3D-RA datasets. (a) The axial MIP image. Volume rendered images based on the segmentation by: (b) the consultant radiologist, (c) Auto-GT and (d) MIP-MP-WTA.

Our method is computationally efficient. In all the experiments, our algorithm takes less than 2 sec and needs $<20$ iterations to segment a 3D-RA image with volume size of around $100 \times 100 \times 100$ voxels on a $2.26 \mathrm{GHz}$ PC with $512 \mathrm{MB}$ RAM.

\section{Conclusions}

This paper has proposed a novel automatic segmentation technique to extract vasculature for 3D rotational angiography (3D-RA). Vascular segmentation of 3D-RA is important in the clinical environment since it can provide 3D information of vasculature before, during and after the treatments. The proposed method is based on a property of maximum intensity projection (MIP), namely, the larger proportion of vessels in the MIP image as compared with the original 3D volume, which can increase the accuracy in the parameter estimation of finite mixture model (FMM) with the expectation maximization (EM) algorithm. The experimental results on 12 3D-RA clinical datasets have indicated that the automatic method can produce more accurate vascular segmentations than the automatic global thresholding method that based on FMM and the EM algorithm. Moreover, our algorithm is capable of producing satisfactory segmentations with a high degree of agreement to the manual segmentations produced by an experienced consultant radiologist. On average, our algorithm takes less than 2sec to segment a 3D-RA dataset in volume size of around $100 \times 100 \times 100$ voxels, which is as computationally efficient as the global thresholding method.

\section{References}

[1] J. Moret, R. Kemkers, J. Op de Beek, R. Koppe, E. Klotz, and M. Grass, “3D rotational angiography: clinical value in endovascular treatment,” Medicamundi, vol. 42, pp. 8--14, 1998.

[2] R. Gan, A. C. S. Chung, W. C. K. Wong, and S. C. H. Yu, "Vascular segmentation in three-dimensional rotational angiography based on maximum intensity projections," in ISBI, to appear, 2004.

[3] S. C. Horii and Y. Kim, Handbook of medical imaging, volume 3: display and PACS. SPIE --- The International Society for Optical Engineering, October 2000, vol. 3.

[4] C. Bishop, Neural networks for pattern recognition. Oxford, Clarendon Press, 1995

[5] D. Wilson and J. Noble, "Segmentation of cerebral vessels and aneurysms from MR angiography data," in IPMI, 1997, pp. 423--428.

[6] A. P. Zijdenbos, B. M. Dawant, R. A. Margolin, and A. C. Palmer, "Morphometric analysis of white matter lesions in MR images: method and validation,” IEEE Trans. on Medical Imaging, vol. 13, no. 4, pp. 716--724, 1994. 